\title{
COMMENT
}

\section{Overcoming linearisation errors in calculating bacterial growth rates}

\author{
Francesc Peters* \\ Institut de Ciències del Mar, CMIMA (CSIC), Passeig Marítim de la Barceloneta 37-49, 08003 Barcelona, Catalunya, Spain
}

When we calculate population dynamics parameters for microbial plankton, we normally jump back and forth between biomass productivities $\left(P^{*}, \Delta\right.$ biomass $\mathrm{vol}^{-1}$ time ${ }^{-1}$ ) and specific growth rates $\left(\mu\right.$, time $\left.^{-1}\right)$ (Ducklow 2000). At each of those steps, there is the possibility to make calculation errors by assuming productivities as rates that are independent from the standing biomass. That is, exponential processes (no matter how close $\mu$ is to zero) may be linearised. In a recent note, Kirchman (2002) has addressed this issue by comparing 2 different equations used to calculate bacterial growth rates from changes in biomass over time. I will readdress this issue and derive somewhat different conclusions.

I will assume that exponential growth is an inherent property of microbial populations. Visualisation of this growth in natural conditions may be masked. As an example, when predators are removed, bacteria often increase exponentially (Weisse \& Scheffel-Möser 1991, del Giorgio et al. 1996). Thus, in situ changes in bacterial biomass may be masked by loss factors that counterbalance growth. However, this does not mean that the microbes are not following the laws of exponential growth. In any case, exponential growth is the basic assumption of the dynamics of planktonic microbial populations and is used in calculations, experiment interpretations and modelling efforts.

I will also assume that the radioactive leucine incorporation technique (Kirchman 1993, Kirchman \& Ducklow 1993) estimates gross bacterial assemblage production. If one further assumes balanced growth and that the bacterial assemblage behaves like a population, the specific bacterial growth rate $(\mu)$ can be calculated. The production $(P)$ after a time $\Delta t$ will be:

$$
P_{\Delta t}=B_{t}-B_{0}
$$

where $B_{0}$ and $B_{t}$ are the initial and final biomass, respectively.

\footnotetext{
*Email: cesc@icm.csic.es
}

Assuming exponential growth,

$$
P_{\Delta t}=B_{0} \mathrm{e}^{\mu \Delta t}-B_{0}
$$

Rearranging,

$$
\mu=\ln \left[\left(P_{\Delta t} / B_{0}\right)+1\right] / \Delta t
$$

One can readily observe from Eqs. (1) \& (3) that $P$ has units of biomass, not of biomass time ${ }^{-1}$.

It is a common mistake to calculate the productivity (in terms of $\mu \mathrm{g} \mathrm{C}^{-1} \mathrm{~d}^{-1}$ or cells $\mathrm{l}^{-1} \mathrm{~d}^{-1}$ ) by dividing the measured production into the incubation time in days. This produces a linearisation error by considering that the productivity is independent from biomass. This concept is also the basis in the note by Kirchman (2002). Consequently, the application of explicitly exponential equations such as Eq. (3) to calculate $\mu$ $\left(d^{-1}\right)$ will be in error. The magnitude of the errors made by such calculations depends on the difference between our incubation time and our reference time (1 d) and on the actual true value of the growth rate (Fig. 1). For all cases where the incubation time is shorter than our reference time we underestimate the true growth rate. The longer the difference between these 2 times, the larger the resulting error. On the other hand, the error decreases with decreasing values for the true growth rate. Taking a true growth rate of $1 \mathrm{~d}^{-1}$ and a leucine incubation time of $1 \mathrm{~h}$, an underestimation of $30 \%$ results when linearisation errors are made in the production calculations which are then used to calculate $\mu$.

The solution to overcome the linearisation errors made when calculating $\mu$ using Eq. (3) is very simple. One just has to plug in the production obtained during the incubation as $P$ and the incubation time as $\Delta t$, without first calculating a daily production. This is the correct way of using Eq. (3). Over the years I have noticed this is rarely done and it is not mentioned in Kirchman (2002).

As the linearisation errors depend on the ratio between our incubation and reference times, we would 


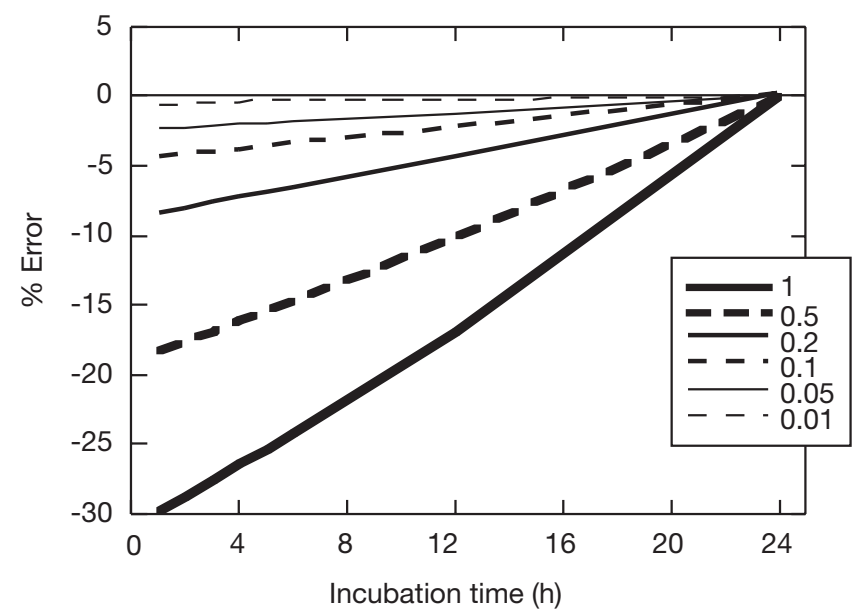

Fig. 1. Linearisation error at incubation times from 1 to $24 \mathrm{~h}$ for different true specific growth rates. Linearisation error was estimated by calculating a daily productivity from the production during the incubation divided by the incubation time in days and then applying Eq. (3) with $\Delta t=1 \mathrm{~d}$. Initial concentration: $5 \times 10^{5}$ bacteria $\mathrm{ml}^{-1}$. Zero percent error line as reference

make no error if our leucine incubation time were $1 \mathrm{~d}$. In this case there would be many other complications with the incubation that would make the technique not viable, but from the point of view of calculations, it would be correct. We could also have a reference time of $1 \mathrm{~h}$ matching an incubation of exactly $1 \mathrm{~h}$. Instead of expressing production rate as $\mu \mathrm{gC}^{-1} \mathrm{~d}^{-1}$ we would use $\mu \mathrm{gC} \mathrm{l}^{-1} \mathrm{~h}^{-1}$. In this case, again, no linearisation error would be produced. On the other hand, if we had an incubation time that exceeded our reference time (say a $3 \mathrm{~h}$ incubation vs a $1 \mathrm{~h}$ reference time), then we would incur in an overestimation of true $\mu$. The error would change sign. But we normally want to refer production to a daily unit and it would be cumbersome (and sometimes impossible) to have exact $1 \mathrm{~h}$ incubations. Thus, we are bound to use Eq. (3) correctly.

The ratio $P / B$, where $P$ is production over a certain time, normalises the production to a defined biomass $B$ (usually initial biomass). It is often expressed as a percentage. One can then see again that both $P$ and $B$ have units of biomass. Moreover, in aquatic systems where bacterial biomass is maintained within relatively narrow bounds, it has been used as an indicator of biomass turnover for the period over which production has been calculated. Since production is more often than not converted to a daily productivity (with correct or incorrect calculations), it is very tempting to replace $P$ by the productivity $P^{*}$. The units turn out to be $\mathrm{d}^{-1}$, which are the units of growth rate.

$$
\mu=P^{*} / B
$$

Eq. (4) is often used to calculate $\mu$, even though there is an obvious linearisation that also produces errors (Fig. 2). Moreover, the biomass used for $B$ is usually the biomass determined at the beginning of that $1 \mathrm{~d}$ period. If changes in biomass occur over this reference time frame then an average biomass (arithmetic or, better, logarithmic) should be used, instead of assuming that $B$ is constant. As an example, if we assume a $\mu$ of $1 \mathrm{~d}^{-1}$ and an initial biomass of $5 \times 10^{5}$ bacteria $\mathrm{ml}^{-1}$, the true production after $1 \mathrm{~d}$ will be $8.59 \times 10^{5}$ bacteria $\mathrm{ml}^{-1}$. Then $P^{*} / B$ will result in $1.72 \mathrm{~d}^{-1}$, a $72 \%$ overestimation with respect to true $\mu$.

However, there is one unexpected result of these calculations. If one has initially made the linearisation error and calculated a daily production rate from the leucine activity data, then applying $P^{*} / B$ paradoxically will result in a lower error than applying Eq. (3) (Fig. 2). This reduction in error occurs for incubation times that are much smaller than the reference time. In fact, the error approaches zero as the incubation time also goes to zero, or in other words, as the measurement of production becomes instantaneous.


Fig. 2. Comparison of (A) calculation of $\mu$ and (B) absolute value of percent error when using Eq. (3) and Eq. (4). Linearisation error estimated as in Fig. 1. True $\mu=1 \mathrm{~d}^{-1}$ 
Additionally, Eq. (4) holds true if we assume the bacterial assemblage to be in steady state. This is one special case in the dynamics of bacterial populations. It occurs when all population loss factors (grazing, virus mortality, autolysis, etc.) match gross production so that net assemblage production is zero. In this situation $\mu=\lambda$ if we think of $\lambda$ as a composite of all specific loss rates. There is no change in the biomass of the population over time, and gross production as well as losses are constant. Thus, we cannot incur linearisation errors. That does not mean that there is no exponential growth, but rather that this growth is exactly matched by an exponential loss.

If we assume that the bacteria in a certain water mass are in steady state, then it is correct to calculate the production rate by dividing the production $P$ by the incubation time $\Delta t$ :

$$
P^{*}=P / \Delta t
$$

Productivity will then be comparable across water masses and over time, provided we assume that the assemblages were in steady state at the time of production estimation. Then Eq. (4) can be used to calculate $\mu$, as it can be derived from

$$
P=\mu B \Delta t
$$

Of course, a previous assumption for the use of these equations is that the data from the leucine technique can be converted into a gross bacterial assemblage production. The technique can certainly not produce an estimate of net bacterial assemblage production, since - if steady state holds true - it would always give a result of zero. However, it could give some value between gross and net assemblage production, and then $\mu$ will accordingly be $\mu^{\prime}$ and not estimate true $\mu$, but this is an issue to address elsewhere.

Do the linearisation errors matter? In the literature, and especially for the open ocean, there are many values of $\mu$ that are fairly small $\left(\leq 0.3 \mathrm{~d}^{-1}\right.$; e.g. Pedros-Alio et al. 1999, Sherr et al. 2001). This implies the existence of long generation times, in comparison to incubation times and to the reference time of $1 \mathrm{~d}$. In these instances linearisation errors are small. In laboratory or field experiments, or in nutrient-rich and/or coastal zones, production tends to be much higher, greatly increasing the linearisation error.

To obtain a better estimate of the magnitude of errors, I analysed some bacterial production data determined by the leucine method. The set contained data from the Southern Ocean (Drake Passage, Bransfield Strait and Gerlache Strait), and from experiments with NW Mediterranean coastal water. I calculated $\mu$ applying Eq. (3) both with and without linearisation error in the determination of production rate. Fairly long incubation times ( 5 to $6 \mathrm{~h}$ ) are needed before the
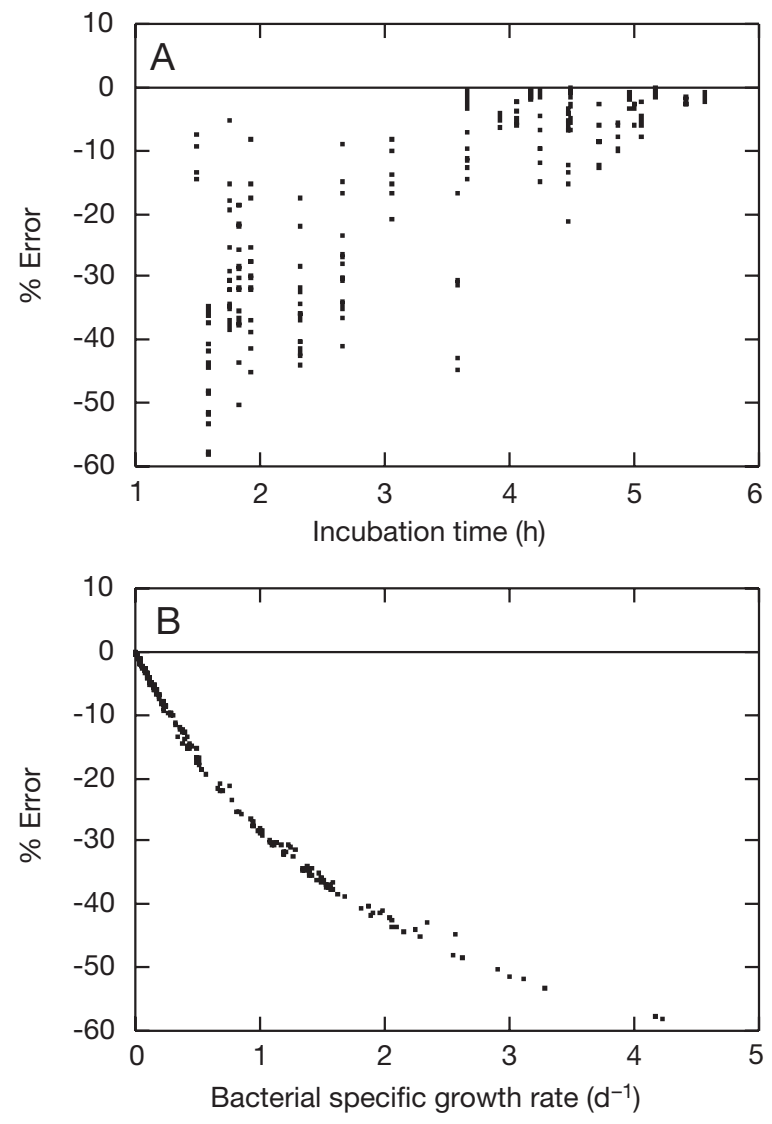

Fig. 3. Error distribution in the calculation of bacterial specific growth rates after linearising the production rate and applying Eq. (3) incorrectly. (A) Errors plotted against incubation times. (B) Errors plotted against $\mu$, as calculated applying Eq. (3) correctly. The dataset $(\mathrm{N}=236)$ includes production measurements from the Southern Ocean and from laboratory experiments with NW Mediterranean water

linearisation error is consistently reduced to less than $10 \%$ (Fig. 3). However, long incubations generate other problems in the technique. In short incubations $(3 \mathrm{~h}$ or less), the range of error is large, from almost non-existent underestimations of $\mu$ to $\geq 50 \%$ (Fig. 3). Here, the errors largely depend on the relative time scale of the true $\mu$ with respect to the incubation time. The absolute value of the error tends to increase with increasing true $\mu$. The shape of the relationship is matched closest by a power function $\left(\mathrm{R}^{2}=0.95\right)$. Overall, Southern Ocean data, which were obtained with longer incubations and had very low values of true $\mu$, showed errors $<21 \%$ with an average of $4.5 \%$. Only 1 station in the Gerlache Strait, with much larger bacterial production, showed errors in the range of 30 to $40 \%$. On the other hand, in the data from experiments with Mediterranean coastal water, where true $\mu$ values are larger, the errors ranged from 5 to $58 \%$ with a mean of $32 \%$. These result from linearisation errors, and may thus be 
significant in coastal zones and in experiments where bacterial production is large.

Measurement errors of $\mu$ based on the leucine production technique may be around $20 \%$. Linearisation errors are often within the magnitude of measurements errors, but in many instances they will be the most important error contrubution in $\mu$ estimates or productivity assessments. More importantly, methods are continuously improving to decrease measurement errors and making additional calculation errors is not warranted when straightforward solutions exist. Propagating such errors unnecessarily deteriorates the quality of large data sets and metadata analyses. It is almost impossible to determine the degree of error due to linearisation mistakes in published data. In the case of bacterial production, one would need the raw data on measurements and incubation times in order to recalculate $\mu$. One would probably also need detailed information about the equations used and the leucine conversion factors.

If published data are suspected of containing linearisation errors before the use of Eq. (3), then I would recommend calculating $\mu$ from Eq. (4), which gives a small error for short incubation times. Otherwise, Eq. (3) should be used, taking care not to incur linearisation errors in calculating daily production rates.

Acknowledgements. Pep Gasol encouraged me to write about the linearisation issue that had bugged me for years and provided the data for Fig. 3. David Bird, Jed Fuhrman, Pep Gasol, Cèlia Marrasé, Carles Pedrós-Alió, Larry Pomeroy, Karel Simek and Dolors Vaqué provided input at some stage of this study. However, any errors in analysis or interpretation

Editorial responsibility: Otto Kinne (Editor),

Oldendorf/Luhe, Germany remain the sole responsibility of the author. This study was funded by the EU project NTAP (EVK3-CT-2000-00022) and is ELOISE contribution no. 350/40.

\section{LITERATURE CITED}

del Giorgio PA, Gasol JM, Vaqué D, Mura S, Agustí S, Duarte CM (1996) Bacterioplankton community structure: protists control net production and the proportion of active bacteria in a coastal marine community. Limnol Oceanogr 41: 1169-1179

Ducklow H (2000) Bacterial production and biomass in the oceans. In: Kirchman DL (ed) Microbial ecology of the oceans. Wiley-Liss, New York, p 85-120

Kirchman DL (1993) Leucine incorporation as a measure of biomass production by heterotrophic bacteria. In: Kemp PF, Sherr BF, Sherr EB, Cole JJ (eds) Handbook of methods in aquatic microbial ecology. Lewis Publishers, Boca Raton, p 509-512

Kirchman DL (2002) Calculating microbial growth rates from data on production and standing stocks. Mar Ecol Prog Ser 233:303-306

Kirchman DL, Ducklow HW (1993) Estimating conversion factors for the thymidine and leucine methods for measuring bacterial production. In: Kemp PF, Sherr BF, Sherr EB, Cole JJ (eds) Handbook of methods in aquatic microbial ecology. Lewis Publishers, Boca Raton, p 513-517

Pedros-Alio C, Calderon-Paz JI, Guixa-Boixereu N, Estrada M, Gasol JM (1999) Bacterioplankton and phytoplankton biomass and production during summer stratification in the northwestern Mediterranean Sea. Deep-Sea Res 46: 985-1019

Sherr EB, Sherr BF, Cowles TJ (2001) Mesoscale variability in bacterial activity in the Northeast Pacific Ocean off Oregon, USA. Aquat Microb Ecol 25:21-30

Weisse T, Scheffel-Möser U (1991) Uncoupling the microbial loop: growth and grazing loss rates of bacteria and heterotrophic nanoflagellates in the North Atlantic. Mar Ecol Prog Ser 71:195-205

Submitted: August 19, 2002; Accepted: November 25, 2002 Proofs received from author(s): December 4, 2002 